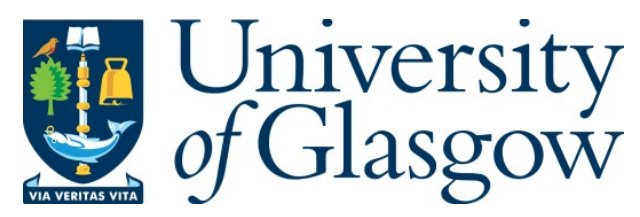

Onireti, O., Imran, A., Imran, M. A., and Tafazolli, R. (2015) Energy efficient inter-frequency small cell discovery in heterogeneous networks. IEEE Transactions on Vehicular Technology, 65(9), pp. 7122-7135.

There may be differences between this version and the published version. You are advised to consult the publisher's version if you wish to cite from it.

http://eprints.gla.ac.uk/132685/

Deposited on: 12 December 2016

Enlighten - Research publications by members of the University of Glasgow http://eprints.gla.ac.uk 


\title{
Energy Efficient Inter-Frequency Small Cell Discovery in Heterogeneous Networks
}

\author{
Oluwakayode Onireti, Member, IEEE, Ali Imran Member, IEEE, \\ Muhammad Ali Imran, Senior Member, IEEE and \\ Rahim Tafazolli, Senior Member, IEEE
}

\begin{abstract}
In this paper, using stochastic geometry, we investigate the average energy efficiency (AEE) of the user terminal (UT) in the uplink of a two-tier heterogeneous network (HetNet), where the two tiers are operated on separate carrier frequencies. In such a deployment, a typical UT must periodically perform inter-frequency small cell discovery (ISCD) process in order to discover small cells in its neighborhood and benefit from the high data rate and traffic offloading opportunity that small cells present. We assume that the base stations (BSs) of each tier and UTs are randomly located and we derive the average ergodic rate and UT power consumption, which are later used for our AEE evaluation. The AEE incorporates the percentage of time a typical UT missed small cell offloading opportunity as a result of the periodicity of the ISCD process. In addition to this, the additional power consumed by the UT due to the ISCD measurement is also included. Moreover, we derive the optimal ISCD periodicity based on the UT's average energy consumption (AEC) and AEE. Our results reveal that ISCD periodicity must be selected with the objective of either minimizing UT's AEC or maximizing UT's AEE.
\end{abstract}

\section{Index Terms}

Heterogeneous cellular network, stochastic geometry, fractional power control, small cell discovery, energy efficiency.

O. Onireti, M. A. Imran and R. Tafazolli are with the Institute for Communication Systems (ICS), Faculty of Engineering \& Physical Sciences, University of Surrey, Guildford GU2 7XH, UK (phone: +44 148368 2423; E-mail: o.s.onireti@ surrey.ac.uk). A. Imran is with Telecommunication Engineering, University of Oklahoma, Tulsa, OK, USA (E-mail: ali.imran@ou.edu). This work was made possible by NPRP grant No. 5-1047-2437 from the Qatar National Research Fund (a member of The Qatar Foundation). 


\section{INTRODUCTION}

To meet the exponentially growing capacity demands, the future of cellular networks is marked by heterogeneous deployments consisting of legacy macro cells with overlaid or underlaid small cells [1]-[7]. Small cell enhancement could either be a scenario where different frequency bands are separately allocated to the small cell and macro cell layers or co-channel deployment scenario, where the small cell and macro cell layers share the same carrier [2]-[4], [8]. It is expected that in the future, small cells will operate on dedicated higher frequency bands, such as $3.5,5$ and beyond $5 \mathrm{GHz}$ bands, where new licensed spectrum is expected to be available [1], [4], [8]. Since small cells have smaller coverage footprint, they do not suffer from the high propagation loss which such band causes to macro cells. Furthermore, cross-tier interference is avoided by operating the small cells on the dedicated higher frequency bands, thus leading to an improvement in spectral efficiency [4]. The use of such bands for small cell can also lead to a significant increase in capacity, since they can offer larger bandwidths. Hence, small cells can provide high data rate to hot spots while also offering traffic offloading opportunity, which can be boosted by incorporating range expansion bias [5], [6].

In the deployments where different frequency bands are separately allocated to the small cell and macro cell layers, user terminals (UTs) connected to the macro cell must periodically scan for suitable small cells in their neighborhood in order to benefit from the high data rate and the traffic offloading opportunity which such offers. This can result in significant energy consumption to the UT. The power limited nature of the UTs is major challenge in enabling truly broadband networks, hence; energy efficient discovery of small cells has been identified by $3 \mathrm{GPP}$ as an important technical issue in carrier-frequency separated deployments [9]. Various inter-frequency small cell discovery (ISCD) mechanisms have been studied in literature. Some of the proposed solutions for enhancing ISCD include: UT speed based measurement triggering [10], [11], relaxed inter-frequency measurement gap [12], proximity based ISCD [11], small cell signal based control measurement and small cell discovery signal in macro layer [3], [13]. A common feature in all the ISCD mechanisms is the periodic inter-frequency scanning and measurement by the UT, which results in significant UT energy consumption.

For a given small cell deployment density and UT speed, low ISCD periodicity (i.e. high scanning frequency) can result in increased small cell offloading opportunity, thus enhancing 
the capacity and coverage. However, this can also lead to higher UT power consumption due to the high scanning frequency. Meanwhile, the UT's transmit power can be reduced as a result of offloading to the small cells where lower transmit power is required due to smaller cell radii. On the other hand, high ISCD periodicity (i.e. low scanning frequency) can lead to the UT missing small cell offloading opportunity, thus resulting in a potential decrease in capacity. Most prior work on ISCD in literature have focused only on the effect of ISCD periodicity on scanning power without evaluating the impact of UT transmit power reduction when offloading to the small cells [10]-[12], [14]. In [14], a mobility aware handover scheme for HetNets consisting of WiMAX and WiFi networks was proposed. In their proposed scheme the UT intelligently selects a subset of the network to be scanned, thus saving UT energy consumption. Mobility based small-cell search has been identified in [10], [11] as an approach that works well within the LTE-A deployment. It has also been shown in [11] that this approach can provide a savings of up to $99 \%$ in UT battery power consumption. Only recently, [15] considered UT transmit power reduction as a result of offloading to the small cell in their evaluation. However, the energy efficiency of this scheme is yet to be investigated. Using stochastic geometry, an analytical framework was proposed in [16] to analyze the trade-off between traffic offloading from the macro cells and the energy consumption of cognitive small cell access points.

In this paper, we investigate the average energy efficiency (AEE) of a typical UT in the uplink of HetNet, where the small cells are deployed on carrier frequency other than that of the serving macro cell and an ISCD scheme is utilized by the UT. The AEE of a communication system is the average amount of bits that can be delivered per joule consumed to do so, i.e. the ratio of the average ergodic rate to the total power consumed [17], [18]. The ergodic rate and the power consumed by a typical UT depend on its association, which could be with either a macro cell or small cell. Hence, the AEE of a typical UT in a HetNet must be obtained by taking the following into consideration: its average power consumption in the macro cell and small cell layers; its average achievable rate in the macro cell or small cell layers; the percentage of time it missed small cell offloading opportunity as a result of the ISCD periodicity and; the additional power it consumes due to ISCD measurement. We model the BS locations as random and drawn from spatial stochastic process, such as homogeneous Poison point process (PPP). In actual deployment, small cells are usually unplanned; hence, they are well modeled by the spatial random process [19]-[22]. On the other hand, modeling macro cell BSs as PPP provide lower 
bounds to the average rate and coverage probability of real deployment [23]. Repulsive point process such as Matern hard core point process (HCPP), which reflect the minimum separation distance between BSs, provides a more realistic model but at the expense analytical tractability [24], [25]. In Section II, we first present the HetNet system model, which incorporates a range extension bias scheme to boost the small cell offloading potential. Next, we present the probability of UT's association to a tier and the probability density function (PDF) of the statistical distance between a typical UT and it serving BS, which later serves as a basis for our derivations. In Section III, we present the ISCD process and its implication in terms of the percentage of time a typical UT missed small cell offloading opportunity. In Section IV, we derive the average UT power consumption and ergodic rate per tier, which are later used in Section V to evaluate its AEE. We derive both the ideal and the realistic AEE of the typical UT in the uplink of the carrier frequency separated HetNet. The ideal AEE is based on an ideal UT association, where the UT associates with the BS (small or macro cell) with the maximum biased received power [6], [22], [26], [27]. On the other hand, the realistic AEE is based on a realistic UT association, where UT association with the small cell is also dependent on the periodicity of the ISCD [11], [12], [15]. In Section VI, we first utilize a polynomial fitting method to approximate the percentage of time the typical UT missed small cell offloading opportunity as a function of ISCD periodicity, for a fixed UT speed and small cell density. Subsequently, by using the approximated function, we derive the average energy consumption (AEC) and AEE optimal ISCD periodicities, for a fixed UT speed and small density. Numerical results are presented in Section VII. Results show that significant savings in the UT's AEC can be achieved by utilizing the optimal ISCD periodicity. Furthermore, ISCD periodicity should be set based on the target objective, which could be towards either AEC minimization or AEE maximization. Finally, conclusions are drawn in Section VIII. A preliminary version of this work has been reported in [28]. Herein, we have considered the interference limited deployment with a cell range extension bias scheme and UT power control.

\section{System ModeL}

We consider a HetNet deployment which is made up of 2 tiers of BSs. The first tier represents macro cell layer while the second tier represents small cell layer. We consider that each tier operates on a different carrier frequency and that each tier is identified by its biasing factor, 
pathloss exponent and, its BSs transmit power and spatial density. The positions of BSs in the $j^{\text {th }}$ tier are modeled according to a homogeneous PPP $\Phi_{j}$ with density $\lambda_{j}$. Furthermore, a fully loaded network with one active uplink user per channel is assumed with the UTs locations approximated by a homogeneous PPP $\Phi^{(\mathrm{u})}$ with density $\lambda^{(\mathrm{u})}$, which is independent of $\left\{\Phi_{j}\right\}_{\{j=1,2\}}$. It is also assumed that the density of the UTs is high enough such that each BS in the network have a least one UT served per channel. We consider that the received signals in the $j^{\text {th }}$ tier are subject to pathloss, which we model using the pathloss exponent $\alpha_{j}$. The random channel variation is modeled as Rayleigh fading with unit mean. We consider that an orthogonal multiple access scheme is utilized within each cell, such that there is no intra-cell interference. Furthermore, each of the BSs in the $j^{\text {th }}$ tier transmit the same power, i.e. $P_{j}$, while the noise power is assumed to be $\sigma^{2}$. In order to evaluate the average UT transmit power, ergodic rate and AEE, we shift all point process such that a typical UT lies at the origin. Regardless of this shift, the homogeneous PPP distribution of the BSs remains preserved.

UT Association: Given that $k \in\{1,2\}$ denotes the index of the tier with which a typical user is associated and $\left|S_{k i}\right|$ is the distance between the typical UT, i.e., the origin and BS $i \in \Phi_{k}$. Also the distance between the typical UT and the nearest BS in the $j^{\text {th }}$ tier is denoted by $D_{j}$. We consider that the UT is associated with a cell based on the maximum biased-received-power (BRP), i.e., the UT associates with the strongest BS in terms of the long-term averaged BRP [22]. The BRPs to the typical UT from the nearest BS in the $j^{\text {th }}$ tier can be expressed as

$$
P_{r, j}=P_{j} L_{0}\left(\frac{D_{j}}{d_{0}}\right)^{-\alpha_{j}} \beta_{j},
$$

where $L_{0}$ denotes the pathloss at a reference distance $d_{0}$ and $\beta_{j}$ is the biasing factor, which is the same for all the BS in the $j^{\text {th }}$ tier. The biasing factor, $\beta_{j}$, can be used to adjust the tier's selection of UTs to allow for effective load balancing. Note that $\left\{\beta_{j}\right\}_{j=1,2}=1$ denotes the conventional cell association, where the UT connects to the BS that offers the highest average received power to the UT.

Distribution of the Distance between UT and Serving BS: It has been shown in [22, Lemma 3] that the probability density function (PDF), $f_{X_{k}}(x)$, of the distance $X_{k}$ between a typical UT and its serving BS in the $k^{\text {th }}$ tier based on the maximum BRP can be expressed as

$$
f_{X_{k}}(x)=\frac{2 \pi \lambda_{k}}{\mathcal{A}_{k}} x \exp \left\{-\pi \sum_{j=1}^{2} \lambda_{j}\left(\widehat{P}_{j} \widehat{\beta}_{j}\right)^{2 / \alpha_{j}} x^{2 / \widehat{\alpha}_{j}}\right\},
$$


where $\mathcal{A}_{k}$, which is defined subsequently in (3), is the idealistic probability of the typical UT associating to the $k^{t h}$ tier.

Idealistic Probability of UT Association to a Tier: In the ideal settings, the UT associates with BSs based on the maximum BRP. In case of UT mobility, handover signaling overhead and other mobility related overheads are not considered. Furthermore, all handover associated time, such as handover preparation time, handover execution time, time to trigger and the ISCD measurement time, are all equal to zero. Hence, in an ideal two-tier HetNet, the idealistic probability that a typical UT is associated with a BS of the $k^{\text {th }}$ tier can be expressed according to [22, Lemma 1] as

$$
\mathcal{A}_{k}=2 \pi \lambda_{k} \int_{0}^{\infty} r \exp \left\{-\pi \sum_{j=1}^{2} \lambda_{j}\left(\widehat{P}_{j} \widehat{\beta}_{j}\right)^{2 / \alpha_{j}} r^{2 / \widehat{\alpha}_{j}}\right\} \mathrm{d} r
$$

where $\widehat{P}_{j} \triangleq \frac{P_{j}}{P_{k}}, \widehat{\beta}_{j} \triangleq \frac{\beta_{j}}{\beta_{k}}, \widehat{\alpha}_{j} \triangleq \frac{\alpha_{j}}{\alpha_{k}}$. It follows that in an ideal UT association, the probability that a typical UT associates with a tier is dependent on the BSs transmit powers, $\left\{P_{j}\right\}_{j=1,2}$, densities $\left\{\lambda_{j}\right\}_{j=1,2}$, and bias factors $\left\{\beta_{j}\right\}_{j=1,2}$. Moreover, $\mathcal{A}_{k}$ can be interpreted as the average fraction of time that a typical UT is connected to the BSs belonging to the $k^{\text {th }}$ tier [26]. Given the total time $T \rightarrow \infty$, the average time that the typical UT spends in the coverage of the macro cell (tier 1) and small cell (tier 2) can be expressed as

$$
\begin{aligned}
& T_{1}=\mathcal{A}_{1} T \quad \text { and } \\
& T_{2}=\mathcal{A}_{2} T,
\end{aligned}
$$

respectively, where $\mathcal{A}_{k}, \forall k=\{1,2\}$ is defined in (3).

Realistic UT Association: In the realistic setting, a typical UT that is connected to the macro cell must periodically scan for suitable inter-frequency small cell (i.e. small cell with higher BRP) before it can discover and offload its traffic (i.e change association) to such small cell. Hence, ISCD scanning and measurements are performed by UTs when associated with the macro cell, at a network or UT specified periodicity. As a result of the scanning periodicity and UT mobility, there exists a fraction of time, $\mathcal{X}$, that the typical UT would miss small cell offloading opportunity. This implies that on the average, the typical UT becomes connected to the macro cell for $\mathcal{X}$ more fraction of time that the small cell provides the maximum BRP. Hence, the average realistic time that the typical UT spends in the macro cell coverage can be expressed 
from (4) as

$$
\tilde{T}_{1}=\mathcal{A}_{1} T+\mathcal{A}_{2} T \mathcal{X}=T\left(\mathcal{A}_{1}+\mathcal{A}_{2} \mathcal{X}\right)
$$

Similarly, the average realistic time that the typical UT spends in the small cell coverage can be expressed as

$$
\tilde{T}_{2}=A_{2} T-\mathcal{A}_{2} T \mathcal{X}=(1-\mathcal{X}) \mathcal{A}_{2} T
$$

\section{InTER-Frequency SMAll CELl Discovery (ISCD)}

A UT connected to the macro cell periodically scans its neighbourhood to discover surrounding small cells. It also performs inter-frequency measurements to ensure that it can connect to another network when it finds a small cell with a higher BRP. The energy consumed for one inter-frequency small cell search can be expressed as

$$
E_{t}=P_{m} T_{m}
$$

where $T_{m}$ is the duration of the measurement and $P_{m}$ is the power consumed by the UT for the measurement. For a given deployment density, $\lambda_{j}$, having a high scanning frequency results in a faster discovery of small cells and hence, increased small cell offloading opportunity, which leads to increase in system level capacity. However, high scanning rate implies an increase in UT's power consumption. On the other hand, reducing the scanning frequency results in the UT missing small cell offloading opportunity, thus, leading to a decrease in system level capacity. Also, the typical UT can significantly reduce its transmit power when connected to the small cells. Consequently, there exists a scanning frequency, $\hat{V}^{\star}$, that achieves optimal performance in terms of average UT energy consumption. If the scanning frequency is less than $\hat{V}^{\star}$, the small cells are not discovered on time, hence excessive UT energy consumption as the UT spends more time in macro cell coverage. On the other hand, excessive energy will be consumed in the search process if the scanning frequency exceed $\hat{V}^{\star}$. The impact of the ISCD frequency, $\hat{V}$, or ISCD periodicity, $V=\frac{1}{\hat{V}}$, can be modelled in terms of the percentage of time the UT missed small cell offloading opportunity, $\mathcal{X}$, as explained in the following.

Consider a typical UT moving according to a random direction mobility model with wrap around [29], [30]. The typical UT moves at a constant speed $\theta$ on [0,1) according to the following mobility pattern: A new direction or orientation is selected from $(0,2 \pi]$ after the UT moves in a particular direction or orientation for a duration $\varsigma$, hence, the selection of the $n^{\text {th }}$ direction 
initializes the $n^{\text {th }}$ movement of the UT. The duration of each movement $\varsigma$ is obtained as the time duration for the UT to move (at a constant speed $\theta$ ) between two farthest points in the HetNet's coverage. In order to obtain $\mathcal{X}$, for a given UT speed, small cell density and ISCD periodicity $V=\frac{1}{\hat{V}}$, we utilize the current 3GPP standard inter-frequency measurement of 40 ms as our benchmark. For the $n^{\text {th }}$ movement with duration $\varsigma$, we estimate the time duration that the UT spends in the coverage of the small cell, based on ISCD periodicity $V$ and the standard inter-frequency measurement of $40 \mathrm{~ms}$, denoted by $\varsigma_{V}^{n}$ and $\varsigma_{40 \mathrm{~ms}}^{n}$, respectively. Hence, the average percentage of time the UT missed small cell offloading opportunity, $\mathcal{X}$, for a fixed UT speed, $\theta$, and small cell density $\lambda_{2}$, can be expressed as

$$
\mathcal{X}=1-\mathbb{E}\left[\frac{\varsigma_{V}^{n}}{\varsigma_{40 \mathrm{~ms}}^{n}}\right],
$$

where $\mathbb{E}$ is the expectation operator.

In Fig. 1, we plot the percentage of time the UT missed small cell offloading opportunity, $\mathcal{X}$, against the ISCD periodicity, $V=\frac{1}{\hat{V}}$ for UT speed, $\theta=3,10,20,30$ and $120 \mathrm{~km} / \mathrm{hr}$, macro cell density $\lambda_{1}=\frac{1}{\pi 400^{2} \mathrm{~m}^{2}}$, small cell density $\lambda_{2}=10 \lambda_{1}$ and $20 \lambda_{1}$, macro cell BS transmit power $P_{1}=46 \mathrm{dBm}$, small cell BS transmit power $P_{2}=26 \mathrm{dBm}$ and pathloss exponent $\alpha_{1}=\alpha_{2}=4$. It is obvious that if the scanning frequency is increased, the UT would miss the small cell offloading opportunity for a lesser time since the discovery process takes place more frequently at the time instance when the typical UT is in the coverage of the new small cell in its path. Also increasing the small cells density results in less likelihood for the typical UT to miss the small cell offloading opportunity. In addition, as the UT speed increases, the UT moves more quickly through the coverage of the small cell, hence an increase in the likelihood that the UT would miss the small cell offloading opportunity. Consequently, as the UT speed increases, the percentage of time that the typical UT missed the small cell offloading opportunity increases for any given ISCD periodicity, as illustrated in Fig. 1

\section{METRICS FOR ENERGy EFFICIENCY EVAluATION}

Let $\mathcal{R}(\mathrm{bit} / \mathrm{s})$ be the achievable rate and $\mathcal{P}_{T}$ be the total power consumed for transmitting data at this rate, then, the AEE can be expressed in terms of the bit-per-Joule as $\mathcal{C}_{J}=\mathcal{R} / \mathcal{P}_{T}$. Hence both the power consumption model and the achievable rate are essential in obtaining the AEE of a communication system. 


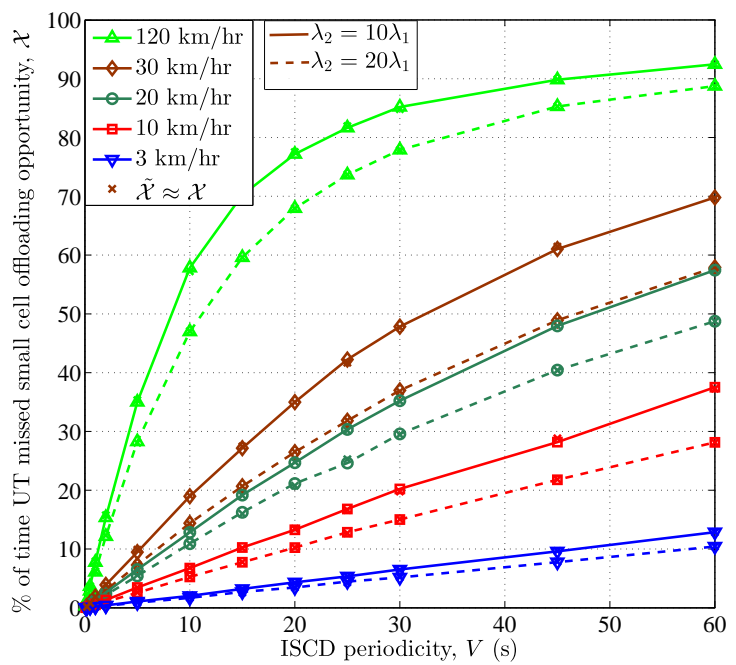

Fig. 1. Percentage of missed small cell offloading opportunity versus small cell discovery periodicity for various UT speed, $\beta_{1}=\beta_{2}=1, \lambda_{1}=\frac{1}{\pi 400^{2} \mathrm{~m}^{2}}, \lambda_{2}=10 \lambda_{1}$ and $20 \lambda_{1}, P_{1}=46 \mathrm{dBm}, P_{2}=26 \mathrm{dBm}$ and $\alpha_{1}=\alpha_{2}=4$.

\section{A. UT Power Consumption Model}

The AEE of a communication system is closely related to its total power consumption. The power consumed by the UT is made up of the transmit power and the additional circuit power incurred during transmission, which is independent of the transmission rate [31], [32]. If we denote the circuit power as $P_{c}$, the overall power consumption of the typical UT at a distance $x$ from its serving BS can be expressed as

$$
\mathcal{P}_{T_{x}}=\Delta P_{x}^{U}+P_{c}
$$

where $P_{x}^{U}$ is the transmission power of the typical UT, $\Delta$ quantifies the UT power amplifier efficiency and it depends on the implementation and design of the transmitter [32].

Average UT Transmit Power in a Tier: Considering that the UT utilizes a distance-dependent fractional power control, hence the transmission power at a distance $x$ to the BS in the $k^{\text {th }}$ tier, $P_{x}^{U}$, is of the form $P_{k}^{0} x^{\alpha_{k} \tau_{k}}$, where $P_{k}^{0}$ is a parameter related to target mean received power (which is user or network specific) in the $k^{t h}$ tier, and $\tau_{k} \in[0,1]$ is the power control factor in the $k^{t h}$ tier. Therefore, as the typical UT moves closer to its associated BS, the transmit power required to achieve the target received signal power at the BS decreases. Hence, having smaller cells, 
where the UT can be closer to their serving BS as opposed to the traditional macro deployment, is expected to yield a reduction in the transmission power. This is an important consideration in power limited devices such as the battery powered mobile devices. The average transmit power of a typical UT in a tier is obtained by averaging $P_{x}^{U}$ over the distance $x$ (i.e., over the $k^{\text {th }}$ tier) and is thus expressed as

$$
\begin{aligned}
P_{k}^{U} & =\mathbb{E}_{x}\left[P_{k}^{0} x^{\alpha_{k} \tau_{k}}\right] \\
& =\int_{0}^{\infty} P_{k}^{0} x^{\alpha_{k} \tau_{k}} f_{X_{k}}(x) \mathrm{d} x \\
& \stackrel{(a)}{=} \frac{2 \pi \lambda_{k} P_{k}^{0}}{\mathcal{A}_{k}} \int_{0}^{\infty} x^{\left(1+\alpha_{k} \tau_{k}\right)} \exp \left\{-\pi \sum_{j=1}^{2} \lambda_{j}\left(\widehat{P}_{j} \widehat{\beta}_{j}\right)^{2 / \alpha_{j}} x^{2 / \widehat{\alpha}_{j}}\right\} \mathrm{d} x
\end{aligned}
$$

where ( $a$ ) follows from (2). If $\alpha_{j}=\alpha, \forall\{j=1,2\}$, the average transmit power of the typical UT over the $k^{\text {th }}$ tier is simplified according to [33, pp. 337] as

$$
P_{k}^{U}=\frac{\pi \lambda_{k} P_{k}^{0} \Gamma\left(1+\frac{\alpha \tau_{k}}{2}\right)}{\mathcal{A}_{k}\left[\pi \sum_{j=1}^{K} \lambda_{j}\left(\widehat{P}_{j} \widehat{\beta}_{j}\right)^{2 / \alpha}\right]^{\left(1+\frac{\alpha \tau_{k}}{2}\right)}}
$$

where $\Gamma$ denotes Gamma function. For the case without power control, i.e. $\tau_{k}=0$, the average transmit power simplifies to $P_{k}^{0}$ in (10) and (11), respectively. Consequently, the average overall power consumption of the UT in the $k^{\text {th }}$ tier can be obtained as

$$
\overline{\mathcal{P}}_{T_{k}}=\Delta P_{k}^{U}+P_{c}
$$

\section{B. Average Ergodic Rate of a Typical UT in a Tier}

The associated signal-to-interference-plus-noise ratio (SINR) at the BS in the $k^{\text {th }}$ tier, which is at a random distance $x$ from the typical UT can be expressed as

$$
\operatorname{SINR}_{k}(x)=\frac{h_{k, 0} P_{k}^{0} x^{\alpha_{k}\left(\tau_{k}-1\right)}}{\sum_{l} h_{k, l} P_{k}^{0}\left|Y_{k, l}\right|^{\alpha_{k} \tau_{k}}\left|V_{k, l}\right|^{-\alpha_{k}}+\sigma^{2}},
$$

where $h_{k, 0}$ is the exponentially distributed channel gain with mean $\mu^{-1}$ from the typical UT, $\left|Y_{k, l}\right|$ is the distance from each interfering UT to their serving BS in the $k^{t h}$ tier, $\left|V_{k, l}\right|$ is the distance from the interfering UT to the BS serving the typical UT in the $k^{\text {th }}$ tier, and $h_{k, l}$ represents the exponentially distributed channel power from $l^{\text {th }}$ interfering UT. Note that there is no inter-tier interference since both tiers operate on separate carrier frequencies. In addition, an orthogonal multiple access is also considered in each cell. 
In order to derive the average ergodic rate of a randomly located UT in the $k^{\text {th }}$ tier, we consider that the UT is associated with the BS with the maximum BRP. We then follow the same approach used in deriving the average UT transmit power in a tier. Firstly, the ergodic uplink rate of a typical UT at a distance $x$ from its serving BS in the $k^{\text {th }}$ tier is obtained. Thereafter, the ergodic uplink rate is then averaged over the distance $x$ (i.e. over the $k^{t h}$ tier). The average ergodic rate of the $k^{\text {th }}$ tier in the uplink channel is thus defined as

$$
\mathcal{R}_{k} \triangleq \mathbb{E}_{x}\left[\mathbb{E}_{\operatorname{SINR}_{k}}\left[\ln \left(1+\operatorname{SINR}_{k}(x)\right)\right]\right]
$$

Contrarily to [34] where the average ergodic rate was obtained based on a fixed minimum distance for the interfering UT, we define the average ergodic rate which is without such limitation in the following theorem.

Theorem IV.1: The average ergodic uplink rate of a typical UT associated with the $k^{\text {th }}$ tier is $\mathcal{R}_{k}=\frac{2 \pi \lambda_{k}}{\mathcal{A}_{k}} \int_{0}^{\infty} \int_{0}^{\infty} x \exp \left\{-\frac{e^{t}-1}{\mathrm{SNR}}-\pi \sum_{j=1}^{2} \lambda_{j}\left(\widehat{P}_{j} \widehat{\beta}_{j}\right)^{2 / \alpha_{j}} x^{2 / \widehat{\alpha}_{j}}\right\} \mathcal{L}_{I_{k}}\left(\mu P_{k}^{0^{-1}} x^{\alpha_{k}\left(1-\tau_{k}\right)}\left(e^{t}-1\right)\right) \mathrm{d} t \mathrm{~d} x^{1}$

where $\mathrm{SNR}=P_{k}^{0} x^{\alpha_{k}\left(\tau_{k}-1\right)} \sigma^{-2}$ and the Laplace transform of the interference to the $k^{\text {th }}$ tier is given by

$$
\begin{aligned}
& \mathcal{L}_{I_{k}}(s)= \\
& \quad \exp \left(-2 \pi \lambda_{k} \int_{x}^{\infty}\left(1-\int_{0}^{\infty} \frac{\mu}{\mu+s P_{k}^{0} y^{\alpha_{k} \tau_{k}} c^{-\alpha_{k}}} \frac{2 \pi \lambda_{k}}{\mathcal{A}_{k}} y \exp \left(-\pi \sum_{j=1}^{2} \lambda_{j}\left(\widehat{P}_{j} \widehat{\beta}_{j}\right)^{\frac{2}{\alpha_{j}}} y^{\frac{2}{\widehat{\alpha}_{j}}}\right) \mathrm{d} y\right) c \mathrm{~d} c\right) .
\end{aligned}
$$

Proof: See Section A of the Appendix.

Note that the average ergodic rate $\mathcal{R}_{k}$ is the average data rate of a typical UT in the $k^{\text {th }}$ tier with only one active UT in each cell. Hence, it also denotes the average cell throughput of the $k^{\text {th }}$ tier when an orthogonal multiple access scheme with round robin scheduling is implemented. Furthermore, the average ergodic rate of a typical randomly located UT in the uplink of a two-tier HetNet can be expressed as

$$
\mathcal{R}=\sum_{k=1}^{2} \mathcal{A}_{k} \mathcal{R}_{k}
$$

\footnotetext{
${ }^{1}$ The effect of the realistic association is captured by combining (15) with some empirical formulas (e.g., [35], [36]).
} 
which simplifies as

$\mathcal{R}=$

$$
\sum_{k=1}^{2} 2 \pi \lambda_{k} \int_{0}^{\infty} \int_{0}^{\infty} x \exp \left\{-\frac{e^{t}-1}{\mathrm{SNR}}-\pi \sum_{j=1}^{2} \lambda_{j}\left(\widehat{P}_{j} \widehat{\beta}_{j}\right)^{2 / \alpha_{j}} x^{2 / \widehat{\alpha}_{j}}\right\} \mathcal{L}_{I_{k}}\left(\mu P_{k}^{0^{-1}} x^{\alpha_{k}\left(1-\tau_{k}\right)}\left(e^{t}-1\right)\right) \mathrm{d} t \mathrm{~d} x .
$$

The ergodic rate expression can be simplified for the noise limited network (noise dominates the interference), which is stated as the following corollary of Theorem IV.1.

Corollary IV.2: The average ergodic rate in the uplink channel of a typical UT associated with the $k^{t h}$ tier for the noise limited $\left(\sigma^{2} \gg I_{k}\right)$ case is given by

$$
\mathcal{R}_{k}=\frac{2 \pi \lambda_{k}}{\mathcal{A}_{k}} \int_{0}^{\infty}-e^{\xi} \operatorname{Ei}(-\xi) x \exp \left\{-\pi \sum_{j=1}^{2} \lambda_{j}\left(\widehat{P}_{j} \widehat{\beta}_{j}\right)^{2 / \alpha_{j}} x^{2 / \widehat{\alpha}_{j}}\right\},
$$

where Ei denotes exponential integral function, $\xi=x^{\alpha_{k}\left(1-\tau_{k}\right)} P_{k}^{0^{-1}} \sigma^{2}$.

\section{ENERgy EFFICIENCy of CARRIER-SEPARATEd HetNet with INTER-Frequency SMall Cell Discovery}

\section{A. Ideal Average Energy Efficiency}

In the previous section we derived generic expressions for the average ergodic rate, $\mathcal{R}_{k}$, and the average power consumption, $\overline{\mathcal{P}}_{T_{k}}$, of the UT in each tier. The ideal AEE in the uplink of HetNet is the ratio of the average bit transmitted by the typical UT to the average energy consumed by the typical UT, while considering the ideal UT association. The average bit transmitted by the typical UT in each tier is obtained from the average ergodic rate and the average time that the typical UT spends in the coverage of each tier, as defined for the ideal association in (4). Given that a typical UT spends an average time $T_{k}$ in the coverage of BSs of the $k^{\text {th }}$ tier, hence the ideal AEE in the uplink of two-tier HetNet can be expressed as

$$
\mathcal{C}_{J}=\frac{\sum_{k=1}^{2} T_{k} \mathcal{R}_{k}}{\sum_{k=1}^{2} T_{k} \overline{\mathcal{P}}_{T_{k}}} \quad(\mathrm{bit} / \mathrm{J})
$$

where $T_{k}, \overline{\mathcal{P}}_{T_{k}}$ and $\mathcal{R}_{k}$ are defined in (4), (12) and (15), respectively. Hence, the ideal AEE in the uplink of HetNet given in (19) can be simplified as

$$
\begin{aligned}
\mathcal{C}_{J} & =\frac{\sum_{k=1}^{2} \mathcal{A}_{k} \mathcal{R}_{k}}{\sum_{k=1}^{2} \mathcal{A}_{k} \overline{\mathcal{P}}_{T_{k}}} \\
& =\frac{\sum_{k=1}^{2} \mathcal{A}_{k} \mathcal{R}_{k}}{\Delta \sum_{k=1}^{2}\left(\mathcal{A}_{k} P_{k}^{U}\right)+P_{c}}
\end{aligned}
$$




\section{B. Realistic Average Energy Efficiency}

As mentioned earlier in Section III, the typical UT consumes additional power $P_{m}$ for each ISCD that it performs when connected to the macro cell. Hence, this additional power must be incorporated into the power consumption model in order to obtain the realistic AEE of the typical UT in the network. It is important to note that apart from the ISCD performed by the UT when connected to the macro cell, which is for exploiting the traffic offloading opportunities available in the small cell, the UT also performs a radio resource management (RRM) interfrequency search when its received signal strength falls below a certain threshold [15]. The RRM inter-frequency search is performed irrespective of the UTs association with either the macro or the small cell with the objective to trigger a handover. This condition arises when the UT is in the cell edge region, where it typically has a lower signal quality. In this work we focus on the additional power consumed by the UT when searching for the small cell with the aim of benefiting from its traffic offloading opportunity, hence we do not consider the RRM inter-frequency search power consumption.

According to the realistic UT association expressions in (5) and (6), the typical UT is connected to the macro cell and small cell for a duration $\tilde{T}_{1}=T\left(\mathcal{A}_{1}+\mathcal{A}_{2} \mathcal{X}\right)$, and $\tilde{T}_{2}=(1-\mathcal{X}) \mathcal{A}_{2} T$, respectively, where $\mathcal{X}$ is obtained empirically. Also, given a fixed ISCD measurement duration $T_{m}$, with ISCD periodicity $V$, the average number of ISCDs that a typical UT experiences in the coverage of the macro cell can be expressed as

$$
\begin{aligned}
N_{I S C D} & =\frac{\tilde{T}_{1}}{T_{m}+V} \\
& =\frac{T\left(\mathcal{A}_{1}+\mathcal{X} \mathcal{A}_{2}\right)}{T_{m}+V} .
\end{aligned}
$$

Hence, the average additional energy consumed by the typical UT as a result of the ISCD measurements in the macro cell coverage can be expressed as

$$
\begin{aligned}
E_{i f m} & =N_{I S C D} T_{m} P_{m} \\
& =\frac{T\left(\mathcal{A}_{1}+\mathcal{X} \mathcal{A}_{2}\right)}{T_{m}+V} T_{m} P_{m},
\end{aligned}
$$

based on the energy consumed for one ISCD measurement, which is given in (7). The AEC of a typical UT in a 2-tier HetNet, $E_{m}$, is thus the sum of the average energy consumed in the first tier (macro coverage), the average energy consumed in searching the small cells, and 
the average energy consumed in the second tier (small cell coverage). Therefore, the AEC of a typical UT can be expressed as

$$
E_{m}=\sum_{k=1}^{2} \tilde{T}_{k} \overline{\mathcal{P}}_{T_{k}}+E_{i f m}
$$

Consequently, the AEE of a typical UT in the uplink of a carrier frequency separated two-tier HetNet, which incorporates the energy consumed for ISCD process, can be expressed as

$$
\mathcal{C}_{J_{C}}=\frac{\sum_{k=1}^{2} \tilde{T}_{k} \mathcal{R}_{k}}{\Delta \sum_{k=1}^{2}\left(\tilde{T}_{k} P_{k}^{U}\right)+T P_{c}+E_{i f m}},
$$

which can be further expressed as

$$
\mathcal{C}_{J_{C}}=\frac{\mathcal{R}_{1}\left(\mathcal{A}_{1}+\mathcal{X} \mathcal{A}_{2}\right)+\mathcal{R}_{2} A_{2}(1-\mathcal{X})}{P_{1}^{U}\left(\mathcal{A}_{1}+\mathcal{X} \mathcal{A}_{2}\right)+P_{2}^{U} \mathcal{A}_{2}(1-\mathcal{X})+P_{c}+\frac{T_{m} P_{m}\left(\mathcal{A}_{1}+\mathcal{X} \mathcal{A}_{2}\right)}{T_{m}+V}}
$$

after substituting for $\tilde{T}_{k}$ and $N_{I S C D}$.

\section{OPTIMAL ISCD PERIODICITY}

In this section, we investigate the optimal ISCD periodicity of a typical UT in the uplink of HetNet based on its AEC and AEE. As discussed earlier, there exists scanning frequencies, $\hat{V}^{\star}$ and $\hat{V}^{\star \star}$, that achieves optimal performance in terms of average UT energy consumption and energy efficiency, respectively. If the scanning frequency is less than $\hat{V}^{\star}$, the small cells will not be discovered on time hence excessive UT energy consumption due to the time duration in macro cell coverage. On the other hand, excessive energy will be consumed in the search process if the scanning frequency exceed $\hat{V}^{\star}$. Similarly, scanning frequency that is less or greater than $\hat{V}^{\star \star}$ will not be energy efficient, since higher scanning frequency means the small cells will be discovered early thus, high capacity at the expense of excessive UT AEC due to scanning. Whereas, a lower scanning frequency means lower capacity, but with savings in UT AEC as a result of scanning. Hence, for scanning frequency higher than $\hat{V}^{\star \star}$, the AEE depreciates due to the excessive power consumption, while the AEE depreciates as a result of the lower rate when the scanning frequency lower than $\hat{V}^{\star \star}$. 
TABLE I

Polynomial Order And Coefficients For Various Deployment Settings

\begin{tabular}{|c|c|c|c|c|c|c|c|c|c|c|}
\hline Speed & \multicolumn{2}{|c|}{$3 \mathrm{~km} / \mathrm{hr}$} & \multicolumn{2}{|c|}{$10 \mathrm{~km} / \mathrm{hr}$} & \multicolumn{2}{|c|}{$20 \mathrm{~km} / \mathrm{hr}$} & \multicolumn{2}{|c|}{$30 \mathrm{~km} / \mathrm{hr}$} & \multicolumn{2}{|c|}{$120 \mathrm{~km} / \mathrm{hr}$} \\
\hline$\lambda_{2}$ & $10 \lambda_{1}$ & $20 \lambda_{1}$ & $10 \lambda_{1}$ & $20 \lambda_{1}$ & $10 \lambda_{1}$ & $20 \lambda_{1}$ & $10 \lambda_{1}$ & $20 \lambda_{1}$ & $10 \lambda_{1}$ & $20 \lambda_{1}$ \\
\hline$N$ & 1 & 1 & 2 & 2 & 2 & 2 & 3 & 3 & 4 & 4 \\
\hline$a_{0}$ & $-1.27 \times 10^{-4}$ & $-1.5 \times 10^{-5}$ & $-2.38 \times 10^{-4}$ & $-3.55 \times 10^{-4}$ & $-1.5 \times 10^{-3}$ & $-7.75 \times 10^{-4}$ & $-2.440 \times 10^{-3}$ & $-9.97 \times 10^{-4}$ & $-4.1 \times 10^{-3}$ & $-2.718 \times 10^{-3}$ \\
\hline$a_{1}$ & $2.148 \times 10^{-3}$ & $1.737 \times 10^{-3}$ & $6.987 \times 10^{-3}$ & $5.378 \times 10^{-3}$ & $1.39 \times 10^{-2}$ & $1.156 \times 10^{-2}$ & $2.1161 \times 10^{-2}$ & $1.5566 \times 10^{-2}$ & $8.54 \times 10^{-2}$ & $6.633 \times 10^{-2}$ \\
\hline$a_{2}$ & - & - & $-1.28 \times 10^{-5}$ & $-1.14 \times 10^{-5}$ & $-7.2 \times 10^{-5}$ & $-5.71 \times 10^{-5}$ & $-1.875 \times 10^{-4}$ & $-1.193 \times 10^{-4}$ & $-3.3 \times 10^{-3}$ & $-2.255 \times 10^{-3}$ \\
\hline$a_{3}$ & - & - & - & - & - & - & $4.8745 \times 10^{-7}$ & $3.4865 \times 10^{-7}$ & $5.9290 \times 10^{-5}$ & $3.7217 \times 10^{-5}$ \\
\hline$a_{4}$ & - & - & - & - & - & - & - & - & $-3.836 \times 10^{-7}$ & $-2.3226 \times 10^{-7}$ \\
\hline
\end{tabular}

A. Approximation of the Percentage of Time a Typical UT Missed Small Cell Offloading Opportunity

In order to obtain the optimal ISCD periodicities in terms of AEC and AEE, i.e, $V^{\star}=\frac{1}{\hat{V}^{\star}}$ and $V^{\star \star}=\frac{1}{\hat{V}^{\star \star}}$, respectively, we must express the percentage of time that a typical UT missed small cell offloading opportunity, i.e. $\mathcal{X}$, as a function of ISCD periodicity $V$. It can be seen in Fig. 1 that $\mathcal{X}$ is a function of the ISCD periodicity, the small cell density and the UT speed. Furthermore, it can be observed that $\mathcal{X}$ can be approximated as a linear function of ISCD periodicity for a fixed UT speed $\theta=3 \mathrm{~km} / \mathrm{hr}$ and small cell densities $\lambda_{2}=10 \lambda_{1}$ and $20 \lambda_{1}$. However, this is not the case for higher UT speed, hence, we generalize the approximation of $\mathcal{X}$ as a function of ISCD periodicity $V$ via a polynomial curve fitting method, for a fixed small cell density and UT speed, as follows

$$
\tilde{\mathcal{X}}(V) \approx \mathcal{X}(V) \approx \sum_{f=0}^{N} a_{f} V^{f}
$$

where $N$ is the order of the polynomial, $a_{f}$ is the $f^{t h}$ polynomial coefficient. The parameter $N$ can be chosen such that the following the mean square error equation is minimized, i.e $\varepsilon_{0} \ll 1$,

$$
\frac{\sum_{\mathbf{V}}\left|\mathcal{X}(V)-\sum_{f=0}^{N} a_{f} V^{f}\right|^{2}}{|\mathbf{V}|} \ll \varepsilon_{0},
$$

where $|\mathbf{V}|$ denotes the cardinality of the test vector $\mathbf{V}$. Table I gives the polynomial order and coefficient for the deployment settings with $\lambda_{2}=10 \lambda_{1}$ and $20 \lambda_{1}$, and $\theta=3,10,20,30,120$ $\mathrm{km} / \mathrm{hr}$. Fig. 1 shows a tight match between the exact percentage of time the UT missed small cell offloading opportunity, $\mathcal{X}$, and its approximation $\tilde{\mathcal{X}}$. 


\section{B. Optimal ISCD Based on Average Energy Consumption}

The average EC expression in (24) can be expressed as a function of the ISCD periodicity as follows

$$
E_{m}(V)=T P_{1}^{U}\left(\mathcal{A}_{1}+\mathcal{X}(V) \mathcal{A}_{2}\right)+T P_{2}^{U} \mathcal{A}_{2}(1-\mathcal{X}(V))+\frac{T T_{m} P_{m}\left(\mathcal{A}_{1}+\mathcal{X}(V) \mathcal{A}_{2}\right)}{T_{m}+V} .
$$

By taking $\mathcal{X}(V) \approx \tilde{\mathcal{X}}(V)$ in $(27), E_{m}(V) \approx \tilde{E}_{m}(V)$, which is clearly differentiable over its domain, such that $\frac{\partial \tilde{E}_{m}(V)}{\partial V}$ can be expressed after simplification as

$$
\frac{\partial \tilde{E}_{m}(V)}{\partial V}=\mathcal{A}_{2}\left(\Delta_{p}\left(T_{m}+V\right)^{2}+T_{m} P_{m}\left(T_{m}+s\right)\right) \frac{\partial \tilde{\mathcal{X}}(V)}{\partial V}-T_{m} P_{m}\left(\mathcal{A}_{1}+\mathcal{A}_{2} \tilde{\mathcal{X}}(V)\right),
$$

where $\Delta_{p}=P_{1}^{U}-P_{2}^{U}$. Let $V^{\star}$ be the solution to the equation $\frac{\partial \tilde{E}_{m}(V)}{\partial V}=0$. Then $\frac{\partial \tilde{E}_{m}(V)}{\partial V} \leq 0$ and $\frac{\partial \tilde{E}_{m}(V)}{\partial V} \geq 0$ for any $V \in\left[0, V^{\star}\right]$ and $V \in\left[V^{\star},+\infty\right]$, respectively, which in turn implies that $\tilde{E}_{m} \approx E_{m}$ decreases over $V \in\left[0, V^{\star}\right]$ and then increases over $V \in\left[V^{\star},+\infty\right]$. Consequently, $E_{m}(V)$ has a unique minimum, which occurs at $V=V^{\star}$. By setting $\frac{\partial \tilde{E}_{m}\left(V=V^{\star}\right)}{\partial V}=0$ and using the approximation of $\mathcal{X}(V)$, for a given speed and small cell density given in Table I in (30), we can obtain $V^{\star}$. For the case where $\mathcal{X}(V)$ is linear, i.e. the polynomial order $N=1$ in (27), the optimal ISCD search based on the AEC can be simplified as

$$
V^{\star}=-T_{m}+\sqrt{\frac{T_{m} P_{m}\left[\mathcal{A}_{2}\left(a_{0}-a_{1} T_{m}\right)+\mathcal{A}_{1}\right]}{\mathcal{A}_{2} a_{1} \Delta_{p}}} .
$$

However, for the case where the polynomial order, $N>1$, we simply use a linear search method such as Newton-Raphson method.

\section{Optimal ISCD Based on UT's Average Energy Efficiency}

The optimal ISCD periodicity in the previous subsection was based on the UT's AEC. In this subsection, we derive the optimal ISCD based on the AEE expression of (26), which can be expressed as a function of the ISCD periodicity as follows

$$
\mathcal{C}_{J_{C}}(V)=\frac{\mathcal{R}_{1}\left(\mathcal{A}_{1}+\mathcal{X}(V) \mathcal{A}_{2}\right)+\mathcal{R}_{2} A_{2}(1-\mathcal{X}(V))}{P_{1}^{U}\left(\mathcal{A}_{1}+\mathcal{X}(V) \mathcal{A}_{2}\right)+\frac{T_{m} P_{m}\left(\mathcal{A}_{1}+\mathcal{X}(V) \mathcal{A}_{2}\right)}{T_{m}+V}+P_{2}^{U} \mathcal{A}_{2}(1-\mathcal{X}(V))} .
$$

Similar to the AEC case, the AEE is differentiable over its domain and the ISCD periodicity that maximizes the AEE, $V^{\star \star}$, can be obtained by setting $\frac{\partial \tilde{\mathcal{C}}_{J_{C}}\left(V=V^{\star \star}\right)}{\partial V}=0$, which simplifies as

$$
\begin{aligned}
\frac{\partial \tilde{\mathcal{C}}_{J_{C}}\left(V=V^{\star \star}\right)}{\partial V} & =0 \\
& =\tilde{E}_{m}(V) \mathcal{A}_{2}\left(\mathcal{R}_{1}-\mathcal{R}_{2}\right) \frac{\partial \tilde{\mathcal{X}}(V)}{\partial V}-\left(\sum_{k=1}^{2} \mathcal{A}_{k} \mathcal{R}_{k}+\left(\mathcal{R}_{1}-\mathcal{R}_{2}\right) \mathcal{A}_{2} \tilde{\mathcal{X}}(V)\right) \frac{\partial \tilde{E}_{m}(V)}{\partial V}
\end{aligned}
$$


Note that the optimal ISCD periodicity based on AEC, i.e. $V^{\star}$, and AEE, i.e. $V^{\star \star}$, are equivalent when the ergodic rate in both tiers are equal, since $\frac{\partial \tilde{\mathcal{C}}_{J_{C}}\left(V=V^{\star \star}\right)}{\partial V}=\frac{\partial \tilde{E}_{m}(V)}{\partial V}$ in (32), when $\mathcal{R}_{1}=\mathcal{R}_{2}$.

\section{NUMERICAL RESULTS AND DisCUSSIONS}

In this section, we present numerical results on the ergodic rate, AEC, AEE and the optimal ISCD periodicity of a typical UT in the uplink of a 2-tier HetNet with both tiers operating on separate carrier frequencies. The system parameters are given in Table II.

TABLE II

SYSTEM PARAMETERS.

\begin{tabular}{c|c|c} 
Parameter & Symbol & Value (units) \\
\hline Bandwidth per tier & $W$ & $20 \mathrm{MHz}$ \\
Macro cell BS density & $\lambda_{1}$ & $\frac{1}{\pi 400^{2} \mathrm{~m}^{2}}$ \\
Small cell BS density & $\lambda_{2}$ & $5 \lambda_{1}, 10 \lambda_{1}, 20 \lambda_{1}$ \\
UT density & $\lambda^{(u)}$ & $100 \lambda_{1}$ \\
Macro cell BS transmit power & $P_{1}$ & $46 \mathrm{dBm}$ \\
Small cell BS transmit power & $P_{2}$ & $26 \mathrm{dBm}$ \\
Small cell Bias factor & $\beta_{2}$ & $0,2,4,6,8,10 \mathrm{~dB}$ \\
UT pathloss compensation factor & $\tau_{1}=\tau_{2}=\tau$ & $0,0.2,0.4,0.6,0.8,1.0$ \\
UT power control parameter & $P_{1}^{0}=P_{2}^{0}=P^{0}$ & $-50 \mathrm{dBm}$ \\
Reference pathloss & $L_{0}$ & $-38.5 \mathrm{~dB}$ \\
Pathloss exponent & $\alpha_{k}$ & $3,3.5,4$ \\
Thermal noise density & $N_{0}$ & $-174 \mathrm{dBm} / \mathrm{Hz}$ \\
\hline
\end{tabular}

\section{A. Achievable rate}

We obtain numerical results for the average ergodic rate (in Theorem IV.1) with respect to the main system parameters; pathloss exponent, power control factor, BS density and bias factor. In Fig. 2, we compare average ergodic rate obtained via simulation with the analytical results. We plot the average ergodic rate as a function of the small cell bias factor, $\beta_{2}$, for small cell density values of $\lambda_{2}=5$, pathloss values $\alpha_{1}=\alpha_{2}=3.5$ and power control factors, $\tau_{1}=\tau_{2}=0.8$ and $\tau_{1}=\tau_{2}=0$. The results in Fig. 2 clearly show that the analytical results provide lower bounds to the average ergodic rate. Furthermore, increasing the small cell bias factor, $\beta_{2}$, leads to a reduction in the average ergodic rate of a typical UT in the small cell whereas the average 


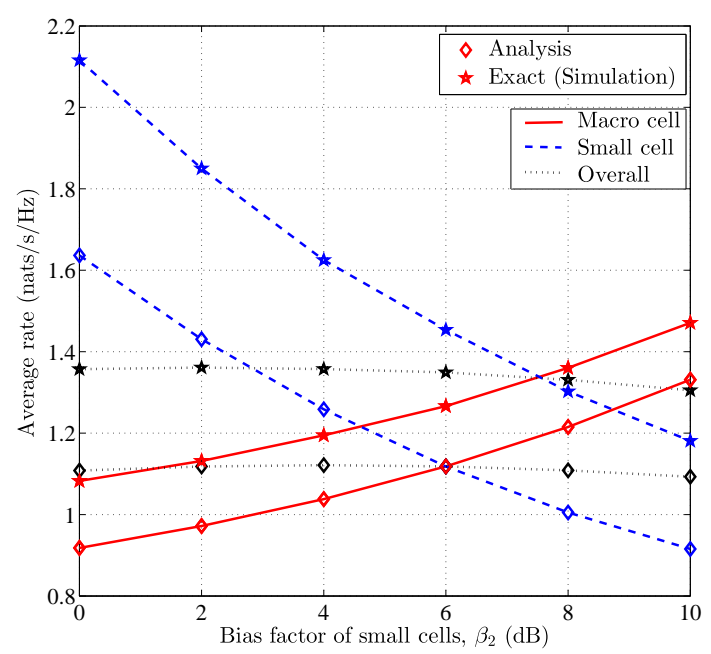

(a) $\tau=0.8$

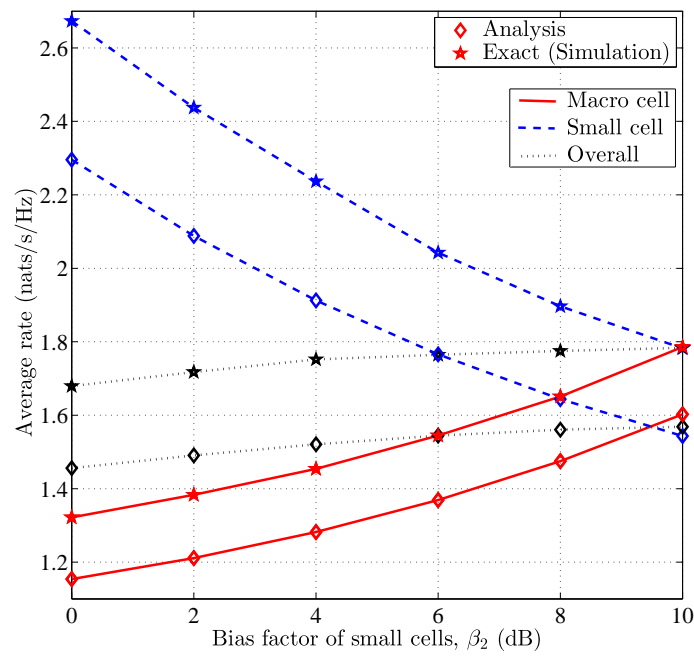

(b) $\tau=0$

Fig. 2. Average ergodic rate for varying bias factor of small cells in a 2 -tier HetNet, $\beta_{1}=1, \lambda_{1}=\frac{1}{\pi 400^{2} \mathrm{~m}^{2}}, \lambda_{2}=$ $5 \lambda_{1}, P_{1}=46 \mathrm{dBm}, P_{2}=26 \mathrm{dBm}$

ergodic rate of the typical UT in the macro cell increases. This is due to the fact that as the small cell bias factor increases, the coverage area of the small cells increases leading to increase in the interference suffered by the typical UT and consequently a reduction in the achievable ergodic rate. As the small cell bias factor increases, more macro UTs with low SINR become associated with the small cell, which degrades the average ergodic rate of the typical UT in the small cell, but improve the rate in the macro cell.

In Fig. 3, using the analytical results, we plot the average ergodic rate of a typical UT as a function of the power control factor, $\tau_{1}=\tau_{2}=\tau$, for pathloss exponents $\left\{\alpha_{1}=3.5, \alpha_{2}=\right.$ $3.5\},\left\{\alpha_{1}=3.5, \alpha_{2}=3\right\}$ and $\left\{\alpha_{1}=3, \alpha_{2}=3.5\right\}$, small cell BS density $\lambda_{2}=10 \lambda_{1}$ and no bias, i.e, $\beta_{1}=\beta_{2}=1$. The results show that the lowest ergodic rate in a tier is achieved by the tier with the lowest pathloss exponent, whereas the contrary holds for the tier with the highest pathloss exponent. This is because the signal from the interfering cells will be stronger with lower pathloss exponent and weaker with higher pathloss exponent i.e., interference decays more slowly as pathloss exponent increases. It can be further observed that the ergodic rate of a typical UT over each tier and over the entire network reduces with increasing power control 


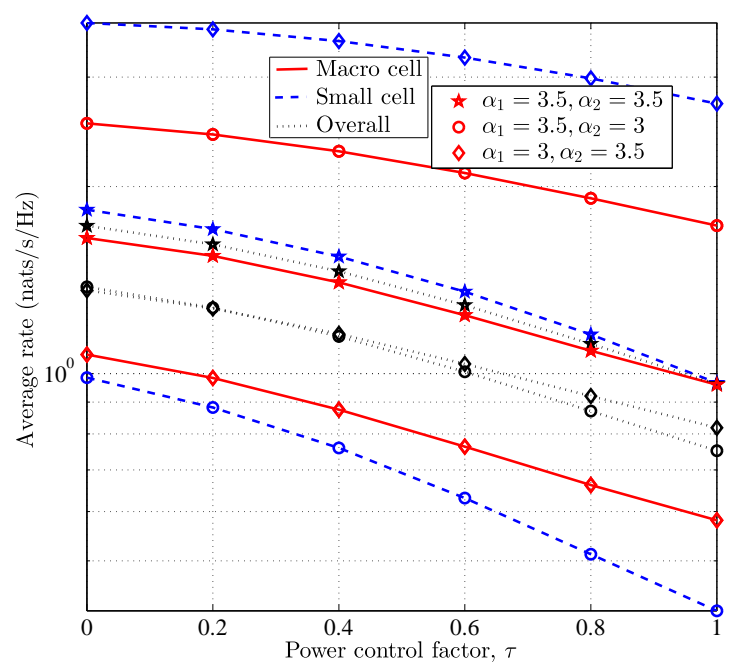

Fig. 3. Average ergodic rate in a 2-tier HetNet as a function of fractional power control parameter $\tau$, for bias factor $\beta_{1}=\beta_{2}=1, \lambda_{1}=\frac{1}{\pi 400^{2} \mathrm{~m}^{2}}, \lambda_{2}=10 \lambda_{1}, P_{1}=46 \mathrm{dBm}$ and $P_{2}=26 \mathrm{dBm}$.

factor $\tau$. Since the obtained rate is for typical UT in the network, the effect of the power control factor on all UTs (i.e., low, medium and high SINR UTs) is combined into a single value. Therefore, the decrease in the average rate as $\tau$ increases is due to the loss in rate of some UTs whose transmit power is reduced, but the effect of this reduction is not overcome on average by the reduction in interference and increased rate by other UTs. Note that this observation was also made for the single tier network in [37].

\section{B. UT Power Consumption}

In Fig. 4, we plot the average UT transmit powers in each tier against the small cell bias factor, $\beta_{2}$, for UT power control, $\tau=1$ and $\tau=0.8$. It can be observed that significant reduction in transmit power is achieved when the UT connects to the small cell compared to when it connects to the macro cell, in the case with full power control, i.e., $\tau=1$. This is as a result of the reduced distance to the BS when typical UT is in the coverage of the small cell, hence a lower transmit power is required to achieve a desired received signal. As the power control factor reduces, the transmit power becomes more independent of the distance between the nodes, hence a reduction in the ratio of the average UT transmit power in the macro cell to that in the small cell. The 


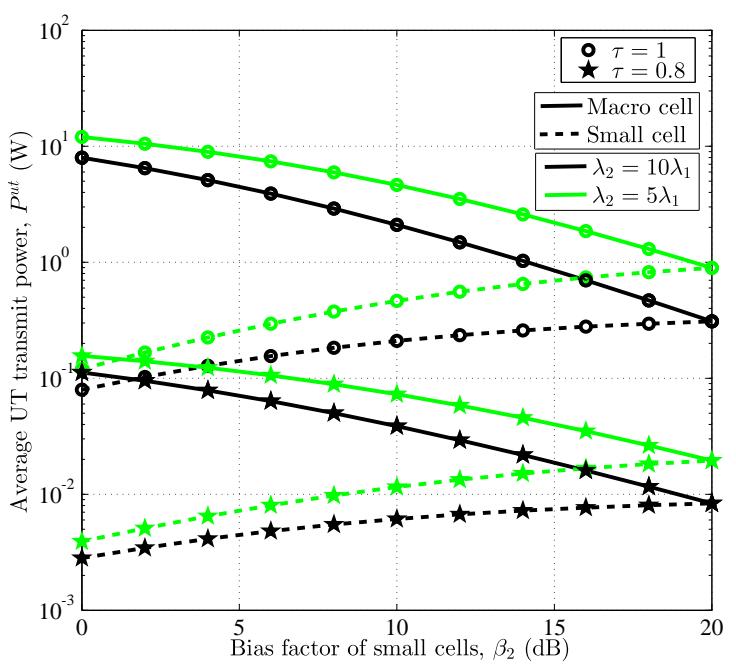

Fig. 4. Average user transmit power for varying bias factor of small cells in a 2 -tier HetNet, $\beta_{1}=1, \lambda_{1}=$ $\frac{1}{\pi 400^{2} \mathrm{~m}^{2}}, \lambda^{(u)}=100 \lambda_{1}, P_{1}=46 \mathrm{dBm}, P_{2}=26 \mathrm{dBm}$, and $\alpha_{1}=\alpha_{2}=3.5$.

result also shows that as expected, the average transmit power in the small cell increases as the small cell bias factor increase, whereas the contrary holds in the macro cell.

\section{Average Energy Efficiency}

The results presented in Sections VII-A and VII-B clearly shows the rate gain and transmit power reduction that is achieved when the UT connects to the small cell of an inter-frequency HetNet. This section presents numerical results on the AEE while considering both the ideal and realistic UT association. Furthermore, the average ergodic rate used in evaluating the AEE is based on the analytical results.

1) Ideal Average Energy Efficiency: In Fig. 5, we plot the ideal AEE, which is based on the ideal UT association against the small cell bias factor. It can be seen that increasing the density of small cells lead to an increase in the UT's AEE in the macro cell, small cell and overall network. Furthermore the UT's AEE performance in the small cell depreciate as the bias factor increases, since the average rate of the typical UT in the small cell decreases while its transmit power increases as the small cell bias factor increases, as shown in Figs. 2 and 4. On the other hand, the performance of the macro cell improves since the contrary occurs. It can 


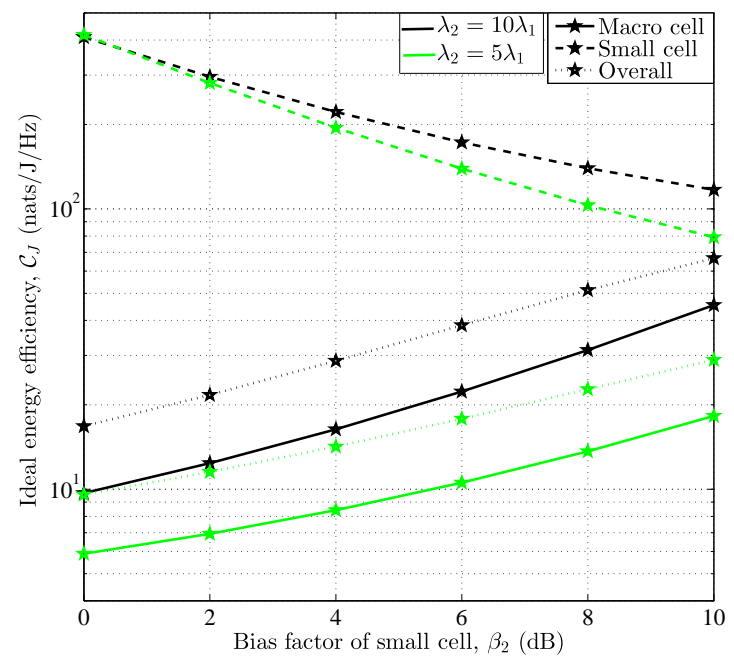

Fig. 5. Ideal AEE for varying bias factor in a 2-tier HetNet, $\beta_{1}=\beta_{2}=1, \lambda_{1}=\frac{1}{\pi 400^{2} \mathrm{~m}^{2}}, \lambda_{2}=5 \lambda_{1}, 10 \lambda_{1}, P_{1}=$ $46 \mathrm{dBm}, P_{2}=26 \mathrm{dBm} \tau=0.8$ and $\alpha_{1}=\alpha_{2}=3.5$.

also be observed that contrary to the overall average ergodic rate in Fig. 2, the overall AEE in a fully loaded network improves with increase in bias factor.

2) Realistic Energy Efficiency: In Fig. 6, we plot the realistic AEE against the small cell discovery periodicity. In the upper graph, typical UT speed $3 \mathrm{~km} / \mathrm{hr}, 20 \mathrm{~km} / \mathrm{hr}$, and $120 \mathrm{~km} / \mathrm{hr}$ are considered for small cell density $\lambda_{2}=10 \lambda_{1}$. The results clearly show that there exists an ISCD periodicity that maximizes the AEE. The lower graph shows the AEE performance for small cell densities, $\lambda_{2}=10 \lambda_{1}, \lambda_{2}=20 \lambda_{1}$ and typical UT speed of $3 \mathrm{~km} / \mathrm{hr}$. As it is expected, increasing the density of the small cells leads to an increase in AEE, since this results in a reduction in the average transmit power of the typical UT coupled with an improvement in the small cell traffic offloading. Furthermore, it can be seen that the optimal ISCD periodicity is dependent on the density of small cells and speed of the typical UTs. For a fixed small cell density, $\lambda_{2}$, a lower small cell discovery periodicity is required to achieve the maximum AEE as the typical UT speed increases. Whereas for a fixed speed of the typical UT, as the small cell density increases, the optimal ISCD periodicity required to achieve the maximum AEE also increases.

Thus this analysis and subsequent determination of optimal ISCD periodicity can pave the 


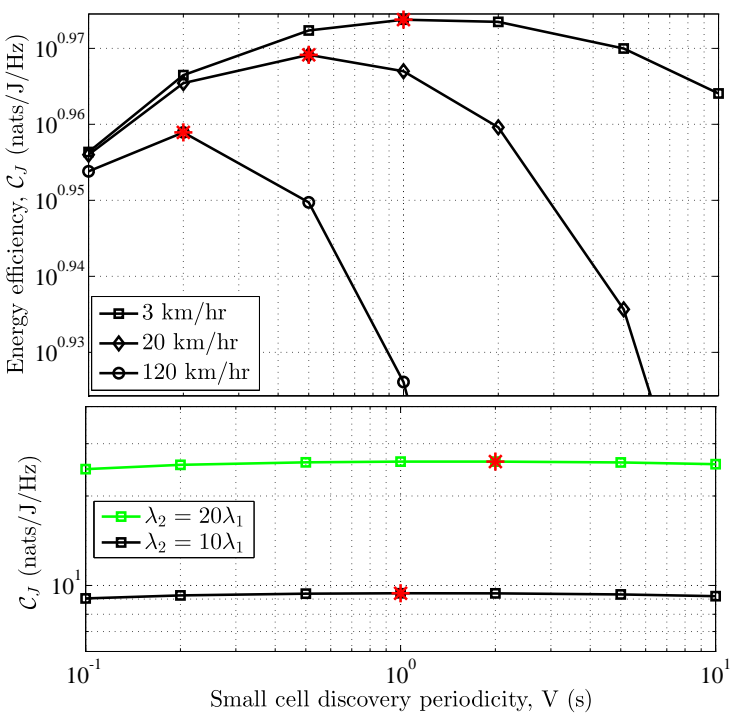

Fig. 6. Realistic AEE for varying small cell discovery periodicity and UT speed, $\beta_{1}=\beta_{2}=1, \lambda_{1}=\frac{1}{\pi 400^{2} \mathrm{~m}^{2}}, \lambda_{2}=$ $10 \lambda_{1}, P_{1}=46 \mathrm{dBm}, P_{2}=26 \mathrm{dBm}, \tau=0.8$ and $\alpha_{1}=\alpha_{2}=4$. The star marker indicates the ISCD periodicity that achieves the optimal AEE.

way towards the design of self organizing network (SON) [38] functions that can adapt the cell discovery periodicity with respect to particular environment (UT speed and small cell density) to achieve optimal AEE performance. Its worth noting that in future HetNets, small cell densities might change impromptu as cell may be switched off and on in order to improve the networks energy efficiency. Hence, the need for such adaptive algorithms that exploits the existence of optimal ISCD for given cell density becomes even stronger.

\section{Optimal ISCD Periodicity}

The results presented in this section are based on a full power control implementation in both tiers, i.e. $\tau_{1}=\tau_{2}=1$. In Fig. 7, we plot the optimal ISCD periodicity for ISCD power consumption $P_{m}$ ranging from $0.01 \mathrm{~W}$ to $2.5 \mathrm{~W}$, average UT transmit power in the macro cell $P_{1}^{U}=1.6114 \mathrm{~W}$, which corresponds to $P_{1}^{0}=-69 \mathrm{dBm}$, UT speed $\theta=3,10$ and $120 \mathrm{~km} / \mathrm{hr}$, and small cell density $\lambda_{2}=10 \lambda_{1}$ and $20 \lambda_{1}$. The average UT transmit power in the small cells with density $\lambda_{2}=10 \lambda_{1}$ and $\lambda_{2}=20 \lambda_{1}$ at $P_{2}^{0}=-50.5 \mathrm{dBm}$ are $1.14 \mathrm{~W}$ and $0.5 \mathrm{~W}$, respectively. The upper graph shows the impact of varying of UT speed on the optimal ISCD periodicity, 


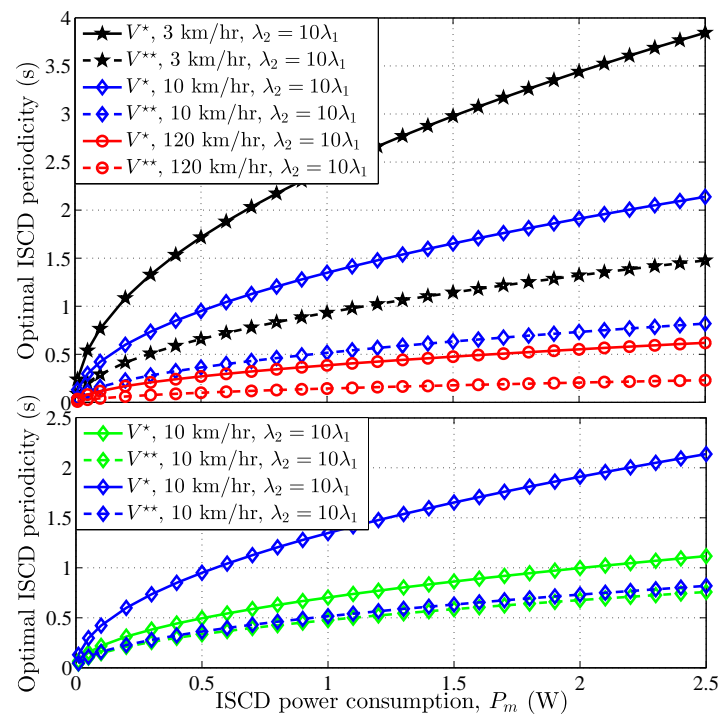

Fig. 7. Optimal ISCD periodicity for various ISCD power consumption, small cell densities, $\lambda_{2}=10 \lambda_{1}, 20 \lambda_{1}$, and UT speed of 3, 10,120 km/hr, UT transmit powers, $P_{1}^{U}=1.6114 \mathrm{~W}$ and $P_{2}^{U}=1.14 \mathrm{~W}$.

while the lower graph shows the impact of varying the small cell density. The upper graph clearly shows that as the UT speed increases, the ISCD periodicities required to achieve optimal AEC and AEE performances reduces. On the other hand, the lower graph shows that increasing the small cell density reduces the ISCD periodicities required to achieve optimal AEC and AEE performances. Furthermore, Fig. 7 clearly shows that increasing the ISCD power results in an increase in the ISCD periodicity required to achieve the optimal performance in terms of both AEC and AEE. Though UT power consumption is lower when UT is connected to the small cell, however, additional power is spent in searching the small cell. Hence increasing the ISCD power implies an increase in the search periodicities required to achieve optimal AEC and AEE performances. Fig. 7 further shows that for a fixed UT transmit power in the small cell, the ISCD periodicity required to achieve optimal AEC performance exceeds the ISCD periodicity required to achieve optimal AEE performance.

In Fig. 8, we plot the average UT power consumption (lower graph) and AEE (upper graph) based on the optimal ISCD periodicity against the ISCD power consumption, $P_{m}$, for small cell density $\lambda_{2}=10 \lambda_{1}$ and UT speed $\theta=3,10$ and $120 \mathrm{~km} / \mathrm{hr}$. As expected, increasing the ISCD power leads to an increase in the average power consumption and a reduction in the AEE. In 


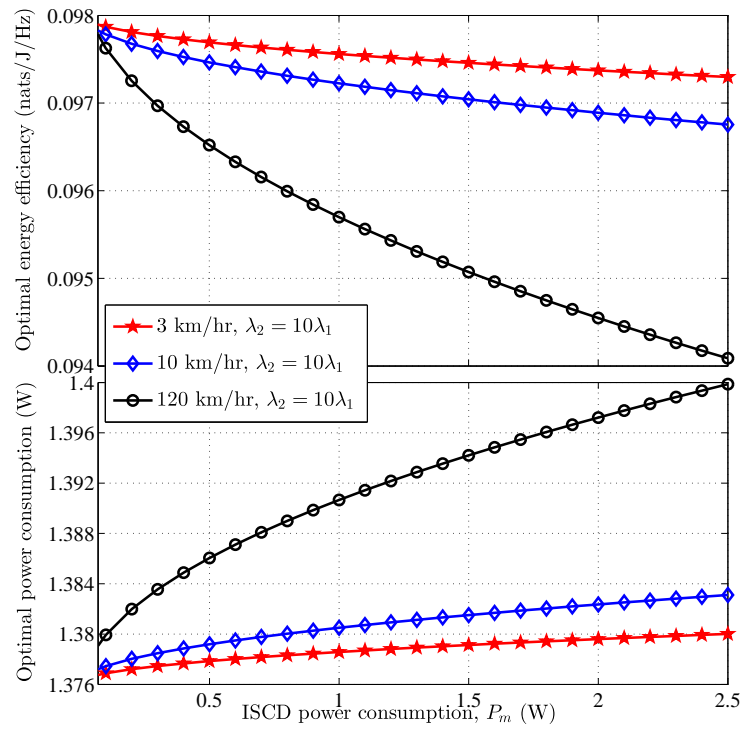

Fig. 8. Average power consumption and AEE based on optimal ISCD periodicity, for small cell density, $\lambda_{2}=10 \lambda_{1}$, and $U T$ speed of $3,10,120 \mathrm{~km} / \mathrm{hr}$, UT transmit powers, $P_{1}^{U}=1.6114 \mathrm{~W}$ and $P_{2}^{U}=1.14 \mathrm{~W}$.

addition, with the same network parameters, a high speed UT is less energy efficient since higher scanning frequency (i.e., lower ISCD periodicity) is required to attain optimal performance.

In Fig. 9, we plot the percentage reduction in AEC (lower graph) and the percentage increase in AEE (upper graph), respectively, that are achieved from using the optimal ISCD periodicity over using sub-optimal ISCD periodicity $V=0.04,0.110$ and $60 \mathrm{~s}$. We plot both graphs for average UT transmit power $P_{2}^{U}$ in the small cell ranging from $0.01 \mathrm{~W}$ to $1.44 \mathrm{~W}$, which corresponds to $P_{2}^{0}$ ranging from $-69.5 \mathrm{dBm}$ to $-49.5 \mathrm{dBm}$, and average UT transmit power in the macro cell $P_{1}^{U}=1.6114 \mathrm{~W}$, which corresponds to $P_{1}^{0}=-69 \mathrm{dBm}$. Fig. 9 shows that significant amount of energy can be saved by adopting the optimal ISCD periodicity especially when there is a large deviation between the optimal and sub-optimal values. For example, the optimal ISCD periodicity for deployment setting with $\lambda_{2}=10 \lambda_{1}, P_{m}=1 \mathrm{~W}, P_{2}^{U}=1.14$ and UT speed of $10 \mathrm{~km} / \mathrm{hr}$ used in Fig. 9 is such that $V^{\star} \wedge V^{\star \star} \in\left[\begin{array}{ll}0.5 & 1.5\end{array}\right] \mathrm{s}$ (as shown in Fig. 7). However, using ISCD periodicity $V=0.04$ and 60 s results in larger difference compared with $V=0.1$ and $10 \mathrm{~s}$, which are more closer to the optimal values.

Since, optimal ISCD periodicity can calculated as function of statistical UT speeds and small cell density only, optimal ISCD periodicity can be maintained in a spatio temporally varying 


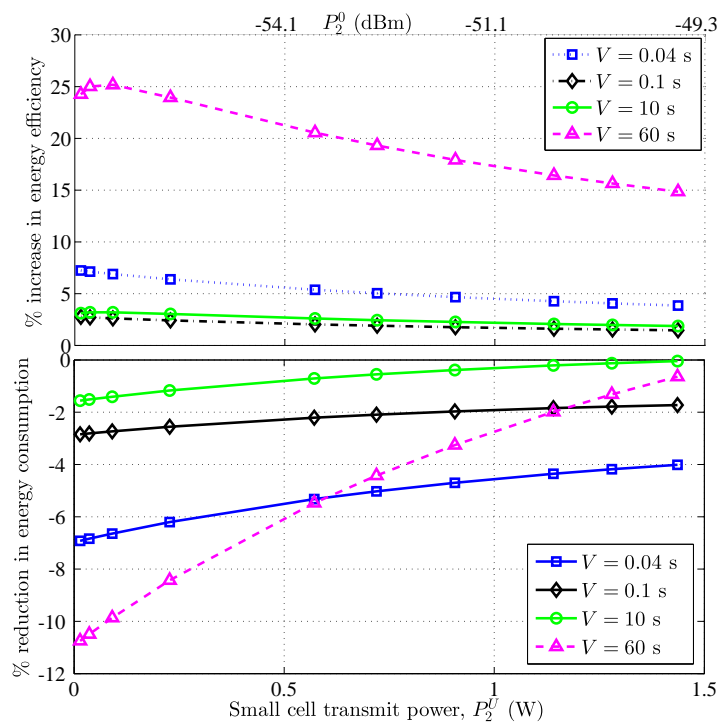

Fig. 9. Percentage reduction in AEC and percentage increase in AEE achieved by using optimal ISCD periodicity over sub-optimal ISCD periodicity, for small cell densities, $\lambda_{2}=10 \lambda_{1}$, UT speed, $\theta=10 \mathrm{~km} / \mathrm{hr}$, ISCD power, $P_{m}=1 \mathrm{~W}$ and UT transmit power, $P_{1}^{U}=1.6114 W\left(P_{1}^{0}=-69 \mathrm{dBm}\right)$.

environment of a HetNet by designing appropriate SON functions, without incurring major overheads in terms of hardware redesign or signaling overheads. As the energy limited nature of UT is one of the major challenges in future broadband networks such as $5 \mathrm{G}$, the significant gain in the AEE of the UT through the implementation of optimal ISCD periodicity can increase the battery life of UT significantly, particularly in ultra-dense HetNets that are being deemed as necessity in $5 \mathrm{G}$ landscape.

\section{CONCLUSION}

In this paper, we have investigated the energy efficiency of the user terminal in the uplink of a carrier frequency separated two-tier heterogeneous network with flexible cell association, also known as biasing. Using Poison point process (PPP) our system model captured the network topology and the design parameters associated with each tier including base station transmit power, density, bias factor, and power control factor. We first derived generic expressions for the average transmit power and average ergodic rate, which were later used in energy efficiency derivation. The energy efficiency expressions are based on the ideal and realistic user terminal 
associations. In the former, user terminals associate with the base station with the maximum biased received signal without considering the overheads required for such association. On the other hand, the latter further incorporates the percentage of time that a typical user terminal missed small cell offloading opportunity as a result of the periodicity of the measurement conducted for small cell discovery. In addition to this, the additional power consumed by the user terminal due to the inter-frequency small cell discovery (ISCD) measurement was also included for the later.

The main findings of this paper can be summarized as follows: Firstly, there exists ISCD periodicity that maximizes the energy efficiency and minimizes the energy consumption when the realistic user terminal association is considered. Secondly, significant savings in the energy consumption of the user terminal can be achieved by using the optimal ISCD periodicity. Lastly, the optimal ISCD periodicity for the user terminal based on energy efficiency always differs from that which is based energy consumption, as long as the average ergodic rate in both tiers differs. Hence, the user terminals ISCD periodicity should be chosen based on the target objectives such as energy consumption minimization or energy efficiency maximization. The findings of this paper can be implemented in real network through self-organizing network functions being already adapted by 3GPP for emerging cellular networks, where the periodicity of the ISCD process can be selected based on the environmental setting to obtain the optimal energy efficiency performance.

Note that randomly distributed network architecture has been presented in this paper. However, future network architectures will be clustered and not randomly distributed. Since accurate modeling of network architecture is crucial, hence a better modeling such as Matérn process with repulsion deserves much attention in future study.

\section{APPENDIX}

\section{A. Proof of Theorem IV.1}

From (14), the average uplink ergodic rate in the $k^{\text {th }}$ tier is

$$
\begin{aligned}
\mathcal{R}_{k} & =\int_{0}^{\infty} \mathbb{E}_{\mathrm{SINR}_{k}}\left[\ln \left(1+\operatorname{SINR}_{k}(x)\right)\right] f_{X_{k}}(x) \mathrm{d} x \\
& =\frac{2 \pi \lambda_{k}}{\mathcal{A}_{k}} \int_{0}^{\infty} \mathbb{E}_{\mathrm{SINR}_{k}}\left[\ln \left(1+\operatorname{SINR}_{k}(x)\right)\right] x \exp \left\{-\pi \sum_{j=1}^{K} \lambda_{j}\left(\widehat{P}_{j} \widehat{\beta}_{j}\right)^{2 / \alpha_{j}} x^{2 / \widehat{\alpha}_{j}}\right\} \mathrm{d} x
\end{aligned}
$$


where $f_{X_{k}}(x)$ is defined in (2). Given that $\mathbb{E}[X]=\int_{0}^{\infty} \mathbb{P}[X>x] \mathrm{d} x$ for $X>0$ hence, we obtain

$$
\begin{aligned}
\mathbb{E}_{\mathrm{SINR}_{k}}\left[\ln \left(1+\operatorname{SINR}_{k}(x)\right)\right] & =\int_{0}^{\infty} \mathbb{P}\left[\ln \left(1+\operatorname{SINR}_{k}(x)\right)>t\right] \mathrm{d} t \\
& =\int_{0}^{\infty} \mathbb{P}\left[\operatorname{SINR}_{k}(x)>e^{t}-1\right] \mathrm{d} t
\end{aligned}
$$

The SINR in (13) can be rewritten as $\gamma(x)=\frac{h_{k}}{P_{0}^{-1} x^{\alpha\left(1-\tau_{k}\right)} Q}$, where $Q=I_{k}+\frac{\sigma^{2}}{L_{0}}$. Hence,

$$
\mathbb{E}_{\mathrm{SINR}_{k}}\left[\ln \left(1+\operatorname{SINR}_{k}(x)\right)\right]=\int_{0}^{\infty} \mathbb{P}\left[h_{k}>P_{0}^{-1} x^{\alpha_{k}\left(1-\tau_{k}\right)} Q\left(e^{t}-1\right)\right] \mathrm{d} t
$$

However,

$$
\begin{aligned}
\mathbb{P}\left[h_{k}>P_{0}^{-1} x^{\alpha_{k}\left(1-\tau_{k}\right)} Q\left(e^{t}-1\right)\right] & =\int_{0}^{\infty} \exp \left[-\mu x^{\alpha_{k}\left(1-\tau_{k}\right)} P_{k}^{0^{-1}}\left(e^{t}-1\right) q\right] f_{Q}(q) \mathrm{d} q \\
& =\mathbb{E}_{Q}\left[\exp \left(-\mu x^{\alpha_{k}\left(1-\tau_{k}\right)} P_{k}^{0^{-1}}\left(e^{t}-1\right) q\right)\right] \\
& =\exp \left(-\frac{e^{t}-1}{\mathrm{SNR}}\right) \mathbb{E}_{I_{k}}\left[\exp \left(-\mu x^{\alpha_{k}\left(1-\tau_{k}\right)} P_{k}^{0^{-1}}\left(e^{t}-1\right) I_{k}\right)\right] \\
& =\exp \left(-\frac{e^{t}-1}{\mathrm{SNR}}\right) \mathcal{L}_{I_{k}}\left(\mu x^{\alpha_{k}\left(1-\tau_{k}\right)} P_{k}^{0^{-1}}\left(e^{t}-1\right)\right)
\end{aligned}
$$

where $\mathrm{SNR}=\frac{P_{k}^{0} x^{\alpha_{k}\left(\tau_{k}-1\right)}}{\sigma^{2}}$ and $\mathcal{L}_{I_{k}}(s)=\mathbb{E}_{I_{k}}\left[e^{-s I_{k}}\right]$ is the laplace transform of $I_{k}$ which simplifies as

$$
\begin{aligned}
L_{I_{k}}(s) & =\mathbb{E}_{I_{k}}\left[\exp \left(-\sum_{z \in \mathcal{Z}_{k}} s P_{k}^{0} Y_{z}^{\alpha_{k} \tau_{k}} V_{z}^{-\alpha_{k}} h_{z}\right)\right] \\
& =\mathbb{E}_{Y_{z}, V_{z}, h_{z}}\left[\prod_{z \in \mathcal{Z}} \exp \left(s P_{k}^{0} Y_{z}^{\alpha_{k} \tau_{k}} V_{z}^{-\alpha_{k}} h_{z}\right)\right] \\
& \stackrel{(a)}{=} \mathbb{E}_{Y_{z}, V_{z}}\left[\prod_{z \in \mathcal{Z}} \mathbb{E}_{h_{z}}\left[\exp \left(s P_{k}^{0} Y_{z}^{\alpha_{k} \tau_{k}} V_{z}^{-\alpha_{k}} h_{z}\right)\right]\right] \\
& \stackrel{(b)}{=} \mathbb{E}_{V_{z}}\left[\prod_{z \in \mathcal{Z}} \mathbb{E}_{Y_{z}}\left[\frac{\mu}{\mu+s P_{k}^{0} Y_{z}^{\alpha_{k} \tau_{k}} V_{z}^{-\alpha_{k}}}\right]\right] \\
& \stackrel{(c)}{=} \exp \left(-2 \pi \lambda_{k} \int_{x}^{\infty}\left(1-\mathbb{E}_{Y_{z}}\left[\frac{\mu}{\mu+s P_{k}^{0} Y_{z}^{\alpha_{k} \tau_{k}} c^{-\alpha_{k}}}\right]\right) c \mathrm{~d} c\right),
\end{aligned}
$$

where $(a)$ is due to the independence of $h_{z},(b)$ follows from the fact that the interference fading power $h_{z} \sim \exp (\mu)$ and $(c)$ is given in [23]. The limits of the integration are from $x$ to $\infty$. Since $x$ is the distance between the typical UT and its serving BS, the closest interferer is at least a distance $x$ from the serving BS of the typical UT. Similar to [37], considering that each BS is randomly located in the Voronoi cell of its corresponding active UT while assuming orthogonal 
multiple access within each cell. Hence, the PDF of the distance between an interfering UT to its serving BS, i.e., $Y_{z}$ can be approximated by the PDF $f_{X_{k}}(x)$ of the distance $X_{k}$ between a typical UT and its serving BS in the $k^{\text {th }}$ tier given in (2). Hence by applying the density of $Y_{z}$, the Laplace transform of the interference in the $k^{\text {th }}$ tier given in (37) can be further expressed as follows

$$
L_{I_{k}}(s)=\exp \left(-2 \pi \lambda_{k} \int_{S_{k}}^{\infty}\left(1-\int_{0}^{\infty} \frac{\mu}{\mu+s P_{k}^{0} y^{\alpha_{k} \tau_{k}} c^{-\alpha_{k}}} \frac{2 \pi \lambda_{k}}{\overline{\mathcal{A}}_{k}} y \exp \left(-\pi \sum_{j=1}^{K} \lambda_{j}\left(\widehat{P}_{j} \widehat{\beta}_{j}\right)^{\frac{2}{\alpha_{j}}} y^{\frac{2}{\alpha_{j}}}\right) \mathrm{d} y\right) c \mathrm{~d} c\right) .
$$

Finally, the average ergodic rate expression in (15) is obtained by substituting (36) into (35) and thereafter substituting the later into (33).

\section{REFERENCES}

[1] Qualcomm Research, "Neighborhood Small Cell for Hyper-Dense Deploments: Taking HetNets to the Next Level," Qualcomm Techology, Inc, Tech. Rep., Feb. 2013.

[2] J.-H. Yun and K. G. Shin, "CTRL: A Self-Organizing Femtocell Management Architecture for Co-Channel Deployment," in MOBICOM, 2010, pp. 61-72.

[3] T. Nakamura, S. Nagata, A. Benjebbour, Y. Kishiyama, T. Hai, S. Xiaodong, Y. Ning, and L. Nan, "Trends in Small Cell Enhancements in LTE Advanced," IEEE Commun. Mag., vol. 51, no. 2, pp. 98-105, Feb. 2013.

[4] H. Ishii, Y. Kishiyama, and H. Takahashi, "A Novel Architecture for LTE-B :C-plane/U-plane Split and Phantom Cell Concept," in IEEE Globecom Workshops (GC Wkshps), Dec. 2012, pp. 624-630.

[5] S. Parkvall, E. Dahlman, G. Jongren, S. Landstrom, and L. Lindbom, "Heterogeneous Network Deployments in LTE: The Soft-cell Approach,” Ericsson Review, Tech. Rep., 2011.

[6] C. de Lima, M. Bennis, and M. Latva-aho, "Statistical Analysis of Self-Organizing Networks with Biased Cell Association and Interference Avoidance," IEEE Trans. Veh. Technol., vol. 62, no. 5, pp. 1950-1961, Jun. 2013.

[7] S. Bu, F. Yu, and H. Yanikomeroglu, "Interference-Aware Energy-Efficient Resource Allocation for OFDMA-Based Heterogeneous Networks With Incomplete Channel State Information,” IEEE Trans. Veh. Technol., vol. 64, no. 3, pp. 1036-1050, Mar. 2015.

[8] A. Mohamed, O. Onireti, M. Imran, A. Imran, and R. Tafazolli, "Control-Data Separation Architecture for Cellular Radio Access Networks: A Survey and Outlook," IEEE Communications Surveys \& Tutorials, Jun. 2015, Early Access.

[9] 3GPP RP-110709, “Study on HetNet Mobility Enhancement for LTE,” Jun. 2011.

[10] A. Prasad, P. Lunden, O. Tirkkonen, and C. Wijting, "Mobility State Based Flexible Inter-Frequency Small Cell Discovery for Heterogeneous Networks," in IEEE 24th International Symposium on Personal Indoor and Mobile Radio Communications (PIMRC), Sept. 2013, pp. 2057-2061.

[11] A. Prasad, O. Tirkkonen, P. Lunden, O. Yilmaz, L. Dalsgaard, and C. Wijting, "Energy-Efficient Inter-Frequency Small Cell Discovery Techniques for LTE-Advanced Heterogeneous Network Deployments," IEEE Commun. Mag., vol. 51, no. 5, pp. 72-81, May 2013.

[12] A. Prasad, P. Lunden, O. Tirkkonen, and C. Wijting, "Energy-Efficient Flexible Inter-Frequency Scanning Mechanism for Enhanced Small Cell Discovery," in IEEE 77th Vehicular Technology Conference (VTC Spring), 2013, Jun. 2013. 
[13] 3GPP TR 36.839, "Mobility Enhancement in Heterogeneous Networks," Sep. 2012, v. 11.0.0.

[14] W.-H. Yang, Y.-C. Wang, Y.-C. Tseng, and B.-S. Lin, "Energy-Efficient Network Selection with Mobility Pattern Awareness in an Integrated WiMAX and WiFi Network," Int'l. J. Commun Sys., vol. 23, no. 2, pp. 213-230, Feb. 2010.

[15] S. Jha, M. Gupta, A. Koc, and R. Vannithamby, "On the Impact of Small Cell Discovery Mechanisms on Device Power Consumption over LTE Networks," in First International Black Sea Conference on Communications and Networking (BlackSeaCom), 2013, Jul. 2013, pp. 116-120.

[16] M. Wildemeersch, T. Quek, C. Slump, and A. Rabbachin, "Cognitive Small Cell Networks: Energy Efficiency and TradeOffs," IEEE Trans. Commun., vol. 9, no. 9, pp. 4016-4029, Sep. 2013.

[17] H. Kwon and T. Birdsall, “Channel capacity in Bits per Joule,” IEEE J. Ocean. Eng., vol. 11, no. 1, pp. 97-99, Jan. 1986.

[18] O. Onireti, F. Heliot, and M. Imran, "On the Energy Efficiency-Spectral Efficiency Trade-Off of Distributed MIMO Systems," IEEE Trans. Commun., vol. 61, no. 9, pp. 3741-3753, Sep. 2013.

[19] Y. Kim, S. Lee, and D. Hong, "Performance Analysis of Two-Tier Femtocell Networks with Outage Constraints," IEEE Trans. Wireless Commun., vol. 9, no. 9, pp. 2695-2700, Sep. 2010.

[20] S. Mukherjee, "UE Coverage in LTE Macro Network with Mixed CSG and Open Access Femto Overlay," in IEEE International Conference on Communications Workshops (ICC), Jun. 2011.

[21] V. Chandrasekhar and J. Andrews, "Spectrum Allocation in Tiered Cellular Networks," IEEE Trans. Commun., vol. 57, no. 10, pp. 3059-3068, Oct. 2009.

[22] H.-S. Jo, Y. J. Sang, P. Xia, and J. Andrews, "Heterogeneous Cellular Networks with Flexible Cell Association: A Comprehensive Downlink SINR Analysis," IEEE Trans. Wireless Commun., vol. 11, no. 10, pp. 3484-3495, Oct. 2012.

[23] J. Andrews, F. Baccelli, and R. Ganti, "A Tractable Approach to Coverage and Rate in Cellular Networks," IEEE Trans. Commun., vol. 59, no. 11, pp. 3122-3134, Nov. 2011.

[24] H. ElSawy, E. Hossain, and M. Haenggi, "Stochastic Geometry for Modeling, Analysis, and Design of Multi-Tier and Cognitive Cellular Wireless Networks: A Survey,” IEEE Commun. Surveys Tuts., vol. 15, no. 3, pp. 996-1019, 2013.

[25] H. ElSawy and E. Hossain, "A Modified Hard Core Point Process for Analysis of Random CSMA Wireless Networks in General Fading Environments," IEEE Transactions on Communications, vol. 61, no. 4, pp. 1520-1534, Apr. 2013.

[26] H. Dhillon, R. Ganti, F. Baccelli, and J. Andrews, "Modeling and Analysis of K-Tier Downlink Heterogeneous Cellular Networks,” IEEE J. Sel. Areas Commun., vol. 30, no. 3, pp. 550-560, Apr. 2012.

[27] M. Di Renzo, A. Guidotti, and G. Corazza, “Average Rate of Downlink Heterogeneous Cellular Networks over Generalized Fading Channels: A Stochastic Geometry Approach,” IEEE Trans. Commun., vol. 61, no. 7, pp. 3050-3071, Jul. 2013.

[28] O. Onireti, A. Imran, M. A. Imran, and R. Tafazolli, “On Energy Efficient Inter-Frequency Small Cell Discovery in Heterogeneous Networks," in IEEE ICC, Jun 2015.

[29] Z. J. Haas, "The Routing Algorithm for the Reconfigurable Wireless Networks?" in Proc. ufICUPC97, San Diego. CA, USA, Oct. 1997.

[30] P. Nain, D. Towsley, B. Liu, and Z. Liu, "Properties of Random Direction Models," in IEEE INFOCOM, Mar. 2005, pp. 1897-2007.

[31] S. Cui, A. Goldsmith, and A. Bahai, "Energy-Efficiency of MIMO and Cooperative MIMO Techniques in Sensor Networks," IEEE J. Sel. Areas Commun., vol. 22, no. 6, pp. 1089-1098, Aug. 2004.

[32] G. Miao, N. Himayat, and G. Y. Li, "Energy-Efficient Link Adaptation in Frequency-Selective Channels," IEEE Transactions on Communications, vol. 58, no. 2, pp. 545-554, Feb. 2010.

[33] I. Gradshteyn and I. Ryzhik, Table of Integrals, Series, and Products, 7th ed. Academic Press, 2007. 
[34] H. ElSawy and E. Hossain, "On stochastic geometry modeling of cellular uplink transmission with truncated channel inversion power control," IEEE Trans. Wireless Commun., vol. 13, no. 8, pp. 4454-4469, 2014.

[35] K. Xu, B. T. Garrison, and K.-C. Wang, "Throughput Modeling for Multi-rate IEEE 802.11 Vehicle-to-infrastructure Networks with Asymmetric Traffic,” in ACM MSWiM, USA, 2011, pp. 299-306.

[36] B. Dusza, C. Ide, and C. Wietfeld, "Interference Aware Throughput Measurements for Mobile WiMAX over Vehicular Radio Channels," in IEEE WCNC Workshops, France, Apr. 2012, pp. 383-387.

[37] T. Novlan, H. Dhillon, and J. Andrews, “Analytical Modeling of Uplink Cellular Networks,” IEEE Trans. Wireless Commun., vol. 12, no. 6, pp. 2669-2679, Jun. 2013.

[38] O. G. Aliu, A. Imran, M. A. Imran, and B. G. Evans, "A Survey of Self Organisation in Future Cellular Networks," IEEE Commun. Surveys Tuts., vol. 15, no. 1, pp. 336-361, 2013. 